\section{Magnetic Nanoparticles Assembled by Micromanipulation of Magnetotactic Bacteria}

A variety of methods have been developed to pattern nanocrystals and assemble them into desired nanostructures. Examples include the formation of singleelectron devices by trapping semiconducting nanoparticles between electrodes and the use of biomineralization by genetically engineered viruses to construct ordered nanostructures. Magnetic nanoparticles can function as singledomain magnets and have applications in spintronics, magnetic memory, and drug delivery. Previously, magnetic nanowires were self-assembled by taking advantage of their large aspect ratios. In addition, magnetic properties have been used to assemble magnetic microstructures. H. Lee, R.M. Westervelt, and coworkers from Harvard University have used microelectromagnets and microfluidics to control the motion of magnetotactic bacteria, which grow intracellular magnetic nanoparticles through a biomineralization process, and have thereby assembled magnetic nanoparticles into ordered structures.

As they reported recently in Nano Letters (Web release date, April 8), Westervelt and co-workers selected the magnetotactic bacteria Magnetospirillum magnetotacticum, which grow intracellular, spherical, magnetite $\left(\mathrm{Fe}_{3} \mathrm{O}_{4}\right)$ nanoparticles contained within a phospholipid membrane. The $50 \mathrm{~nm}$ nanoparticles, which have a narrow size distribution and act as single-domain permanent magnets, are typically assembled into single or multiple chains that are anchored within the cell. Using either optical or electron-beam lithography, the researchers fabricated two types of microelectromagnets on a $\mathrm{Si} / \mathrm{SiO}_{2}$ substrate: a ring trap, which consists of a wire following a circular path and an insulating layer; and a matrix, which consists of two perpendicular arrays of straight conducting wires that are separated and capped with insulating layers.

The researchers used soft lithography to fabricate the microfluidic chamber from poly(dimethylsiloxane) with dimensions chosen to minimize viscous drag on the bacteria. After treating both the electromagnets and the chamber with $\mathrm{O}_{2}$ plasma to render the surfaces hydrophilic, the researchers conformally sealed the chamber on top of the microelectromagnets. Currents were chosen such that the magnitude of the magnetic field was about $0.1 \mathrm{~T}$, which enabled stable trapping of the bacterium, each of which have a magnetic moment of about $10^{-15} \mathrm{~A} \mathrm{~m}^{2}$,

\section{Carbon Nanotubes Used as Nanoscale Mass Conveyors}

The very concept of nanotechnology necessitates nanofabrication, including the manipulation and transport of atoms and molecules. Scanning probe techniques have already been used to manipulate atoms and molecules on surfaces, but they cannot efficiently move atoms from a distant reservoir to a work area. Carbon nanotubes have been proposed as conduits for transporting nanoscale volumes of materials because of their hollow cores and large aspect ratios. B.C. Regan, A. Zettl, and their co-workers at the University of California, Berkeley, and Lawrence Berkeley National Laboratory have demonstrated controllable mass transport along carbon nanotubes using indium as the mass transport species. Controllable mass transport of gold, platinum, tin, and tin indium alloy was also demonstrated.

As reported in the April 29 issue of Nature (p. 924), indium metal was thermally evaporated onto a boule of arc-grown multiwalled carbon nanotubes (MWNTs) decorating them with isolated In nanocrystals. The boule was then placed in a transmission electron microscope (TEM) that included a nanomanipulator. An individual nanotube was approached using a tungsten tip mounted on the nanomanipulator, and a contact was established with the free end of the nanotube (see Figure). An electric current was induced in the nanotube by applying voltage between the tip and the sample holder, resulting in Joule heating. The temperature could be manipulated by varying voltage. TEM video imaging was used to monitor the indium nanoparticles on the nanotube. Passing current through the nanotube resulted in the transport of In toward the cathode with a near unity correlation for mass transfer. This was observed as shrinkage of particles on the anode side accompanied by growth of particles on the cathode side.

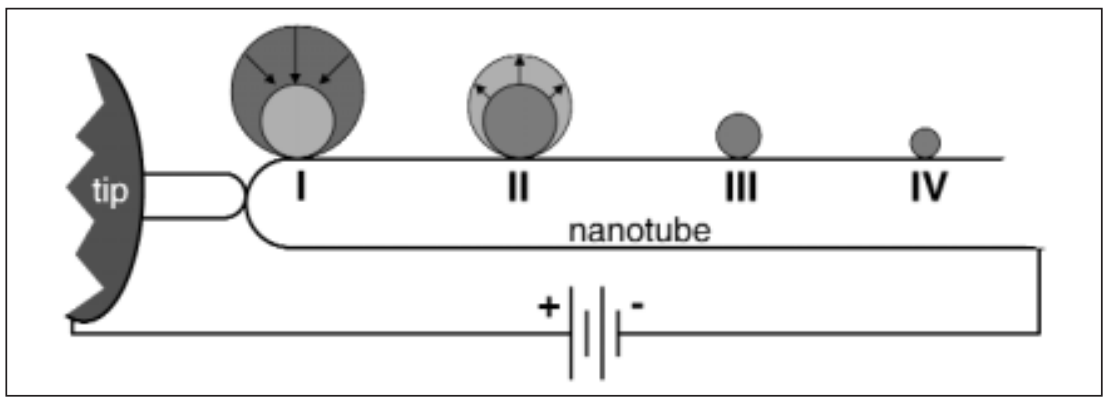

Figure. Schematic illustration of the experimental setup. Four indium particles (labeled I-IV) distributed on a nanotube substrate grow and shrink as current is passed from the tungsten tip through the nanotube.

The transfer of In was demonstrated over a nanotube length of more than $2 \mu \mathrm{m}$. In addition, reversal of the voltage resulted in a reversal of the mass transport direction, with depleted particles reforming. The voltage gradient rather than the thermal gradient was seen to dictate the direction of the mass transport. The application of a square-wave function to the voltage was shown to precisely control the transport of In. According to the research team, this approach has the potential for mass delivery on an atom-by-atom basis for the tailored formation of a final preselected particle size, which has significant implications for nanoscale applications including the use of a nanotube as a "nano-soldering iron."

The researchers have developed a quantitative model for the observed nanoscale mass transfer phenomenon, using the analogy of mass conveyor belts for the transport and mass reservoirs. Plots of mass versus time for various particles along a nanotube were shown to agree well with experimental data points. The mass change rates were found to be linear, indicating that surface energies, which change with particle size, do not play a significant role in driving the transport process down to at least the femtogram level. The exact nature of the driving mechanism, however, has yet to be determined. A comparison was made to indium electromigration toward the cathode on silicon surfaces. The study demonstrates that it is possible to precisely control mass transport at the nanoscale; this bodes well for future nanotechnology applications.

GOPAL RAO 